\title{
Primary chest wall lymphoma with no history of tuberculous pyothorax: Diagnosis and treatment
}

\author{
Abbas Tabatabai, MD, ${ }^{\mathrm{a}}$ Mozaffar Hashemi, MD, ${ }^{\mathrm{a}}$ Mojtaba Ahmadinejad, MD, ${ }^{\mathrm{b}}$ Saeid Haghdani, ${ }^{\mathrm{c}}$ Mahmoud Sajjadi, \\ Parvin Mahzouni, MD, ${ }^{\mathrm{d}}$ and Ali Hekmatnia, $\mathrm{MD}^{\mathrm{e}}$
}

\begin{abstract}
Objective: Primary chest wall neoplasm represents only 5\% of chest wall neoplasm and among them primary chest wall lymphoma is uncommon.

Methods: A 28-year-old man had no history of tuberculous pyothorax or artificial pneumothorax therapy but did have a 4-month history of dyspnea, fever, chills, and night sweats. On physical examination, a mass about $10 \times$ $10 \mathrm{~cm}$ was noted on the anterior chest wall on the right side, and computed tomographic scan demonstrated that it originated from the pleural wall. A 42-year-old man was admitted with intermittent left hemithoracic pain from about 6 months before his visit. A $5 \times 5$-cm tender mass in the posterior wall of the left hemithorax was palpated. Computed tomography showed mild plural effusion and erosion in the posterior segment of the left ninth rib. Surgery was performed for histologic diagnosis.
\end{abstract}

Results: With the diagnosis of large B cell lymphoma, chemotherapy was prescribed for the first patient, and the patient has been in complete remission for more than 5 months. For the second patient, the left ninth rib along with the originated mass was completely resected and chemotherapy was prescribed. The patient has been in complete remission for more than 8 months.

Conclusion: Treatment of primary chest wall lymphoma was not clear and various treatment strategies were considered. Remission of considerable duration in our patients leads us to suggest that surgery followed by adjuvant chemotherapy can provide a reasonable outcome in patients in whom the chest wall lymphoma is the only site of disease.

The chest wall is the origin for a wide variety of malignant neoplasms, which include primary neoplasm, adjacent neoplasm with local invasion, and metastatic lesions. Primary chest wall neoplasm in comparison with the other two groups of chest wall neoplasm mentioned herein is rare, representing only 5\%. ${ }^{1,2}$ Among primary chest wall neoplasms, chest wall lymphoma is uncommon. To date, only a few cases of primary malignant lymphoma arising from the pleura or the rib with no history of tuberculous pyothorax and artificial pneumothorax therapy have been reported, most of which occurred in Japan. ${ }^{3,4}$ Treatment of primary chest wall lymphoma was not clear and various treatment strategies were considered. Surgery followed by adjuvant chemotherapy can provide a satisfactory outcome in patients in whom the chest wall lymphoma is the only site of disease. ${ }^{5}$

Herein we report the clinical and pathologic characteristics as well as treatment strategy of non-Hodgkin lymphoma in 2 patients with a solitary chest wall mass.

From the Departments of Surgery, ${ }^{a}$ Pathology, ${ }^{\mathrm{d}}$ and Radiology, ${ }^{\mathrm{e}}$ Alzahra University Hospital, Isfahan University of Medical Sciences, Isfahan, Iran; the Department of Surgery, Shohada Hospital, Lorestan University of Medical Science, Khorram Abad, Iran ${ }^{\text {b }}$ and the School of Medicine, Isfahan University of Medical Sciences, Isfahan, Iran, Medical Student Research Committee. ${ }^{\mathrm{c}}$

Received for publication Jan 14, 2008; accepted for publication March 3, 2008.

Address for reprints: Saeid Haghdani, Poursina Hakim Research Institute, Post Office Box 81588-44771, Isfahan, Iran (E-mail: saeed_haghdani@yahoo.com).

J Thorac Cardiovasc Surg 2008;136:1472-5

$0022-5223 / \$ 34.00$

Copyright (C) 2008 by The American Association for Thoracic Surgery doi:10.1016/j.jtcvs.2008.03.060

\section{CLINICAL SUMMARIES}

PATIENT 1. A 28-year-old nonsmoking man had a 4-month history of dyspnea, fever, chills, and night sweats. He had been known to have end-stage renal disease for 7 years and rejected kidney transplantation. He had no history of tuberculous pyothorax, artificial pneumothorax therapy, or other remarkable medical disease. On physical examination, a mass of about $10 \times 10 \mathrm{~cm}$ was palpated on the anterior wall of chest on the right side.

Significant laboratory findings were as follows: hemoglobin $6.3 \mathrm{~g} / \mathrm{dL}$, erythrocyte sedimentation rate $60 \mathrm{~mm} / \mathrm{h}$, and serum C-reactive protein $3+$. Chest radiograph revealed a homogeneous chest wall mass and computed tomography (CT) demonstrated a tumor in the pleural wall (Figure 1). Excisional biopsy was performed for histologic diagnosis.

Histologic study showed diffuse infiltration of large lymphocytes with vesicular and prominent nucleoli and relatively abundant cytoplasm. Mitotic activity was high and an area of necrosis was seen. Immunohistochemical staining of the specimen was positive for leukocyte common antigen, CD20, and Ki67 and negative for CD3, vimentin, desmin, chromogranin, neuron specific enolase, CD99, and actin.

Under the diagnosis of large B cell lymphoma, chemotherapy was prescribed for the patient. The patient has been in complete remission for more than 5 months.

PATIENT 2. A 42-year-old Iranian man was admitted with a chief symptom of intermittent left hemithoracic pain, which had begun about 6 months before his visit and had become chronic in the past 2 months. Pain radiated to behind 


\section{Abbreviations and Acronyms}

$\mathrm{CT}=$ computed tomography

PLB $=$ primary lymphoma of the bone

the ribs in the anterior and transverse process of spine in the back. He had a history of spinal cord epandimoma 13 years earlier. He had smoking history of 20 packs per year. Physical examination showed a $5 \times 5$-cm tender mass in the posterior wall of left hemithorax, which extended between the sixth and tenth thoracic vertebrae. He did not have lymphadenopathy or hepatosplenomegaly.

Laboratory examination of the blood revealed a hemoglobin value of $12.2 \mathrm{~g} / \mathrm{dL}$ and a white blood cell of about 9900 . CT demonstrated mild plural effusion and also erosion in the posterior segment of left ninth rib (Figure 2). Bone scan confirmed a lesion in the posterior arch of left ninth rib. Surgery was performed for histologic diagnosis. With the patient under general anesthesia, a mass originating from the ninth rib was resected. Histologic study showed diffuse infiltration of a large lymphocyte with vesicular and prominent nucleoli and relatively abundant cytoplasm. Mitotic activity was high and an area of necrosis was present. Immunohistochemical staining of the specimen was positive for leukocyte common antigen, CD20, Ki67, and CD20 and negative for CD3 and vimentin. The tumor was diagnosed as large B cell lymphoma (Figure 3).

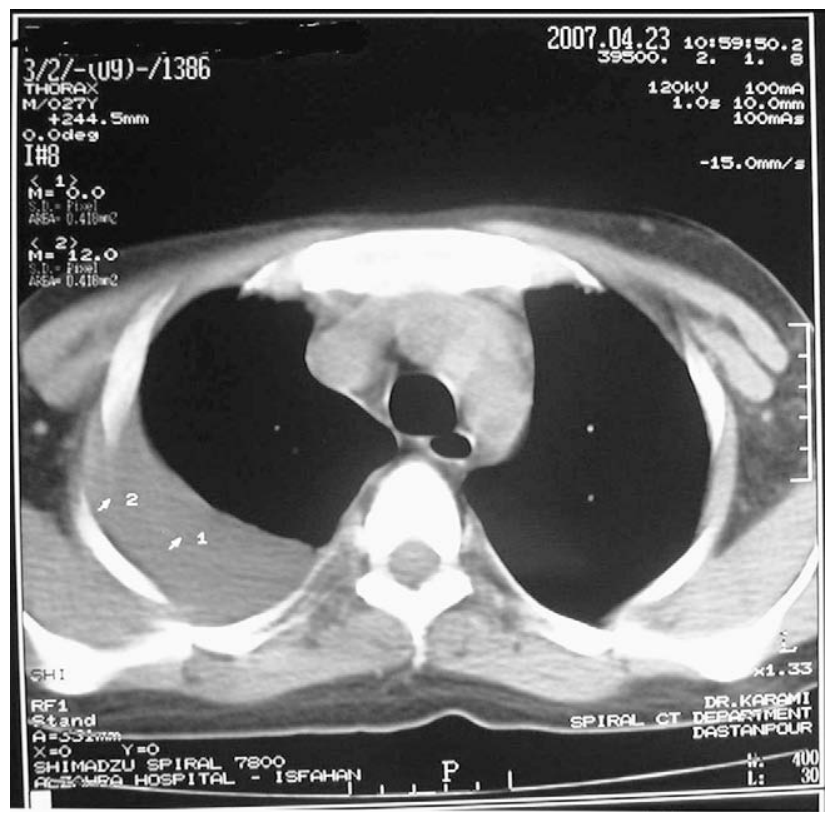

FIGURE 1. CT scan without contrast: Soft tissue mass lesion in posterior aspect of right chest wall with extension into right hemithorax accompanied by pleural thickening and effusion.

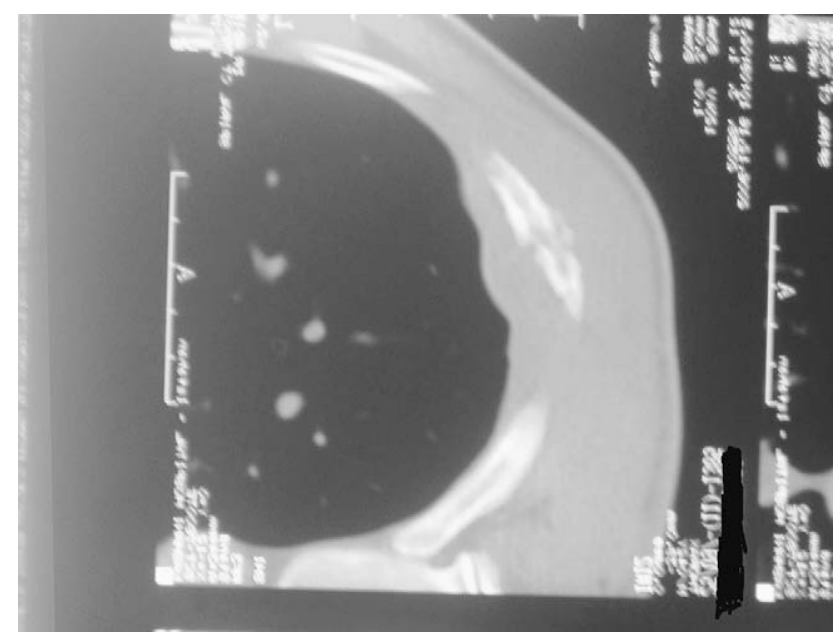

FIGURE 2. CT scan demonstrated an expansile bony lytic destructive lesion in the posterior arch of the left rib with invasion into the left pleural space and accompanied by a posterior soft tissue component.

A biopsy specimen of the bone marrow showed no evidence of lymphoma. The left ninth rib along with the originatal mass was completely resected and chemotherapy was prescribed for the patient. The patient has been in complete remission for more than 8 months after the operation (Figures 1 to 4 ).

\section{DISCUSSION}

Primary malignant lymphoma originating from the pleura is uncommon, comprising only $2.4 \%$ of primary chest wall tumors. ${ }^{6}$ Most pleural lymphomas develop in patients with chronic tuberculous pyothorax or a history of artificial pneumothorax. Most cases of this disease are reported in Japan, 3,7

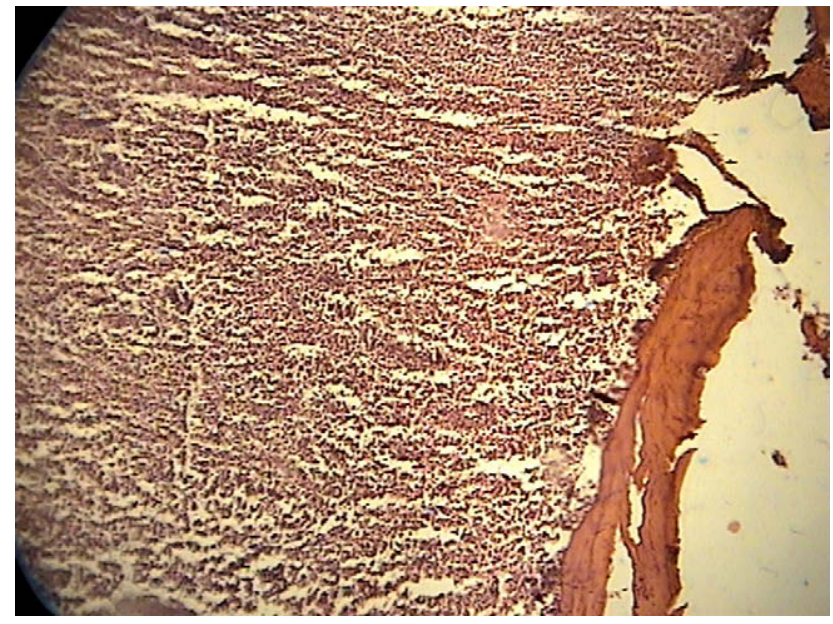

FIGURE 3. Histologic study shows diffuse infiltration of large lymphocytes with vesicular and prominent nucleoli and relatively abundant cytoplasm, which is compatible with large B cell lymphoma. 


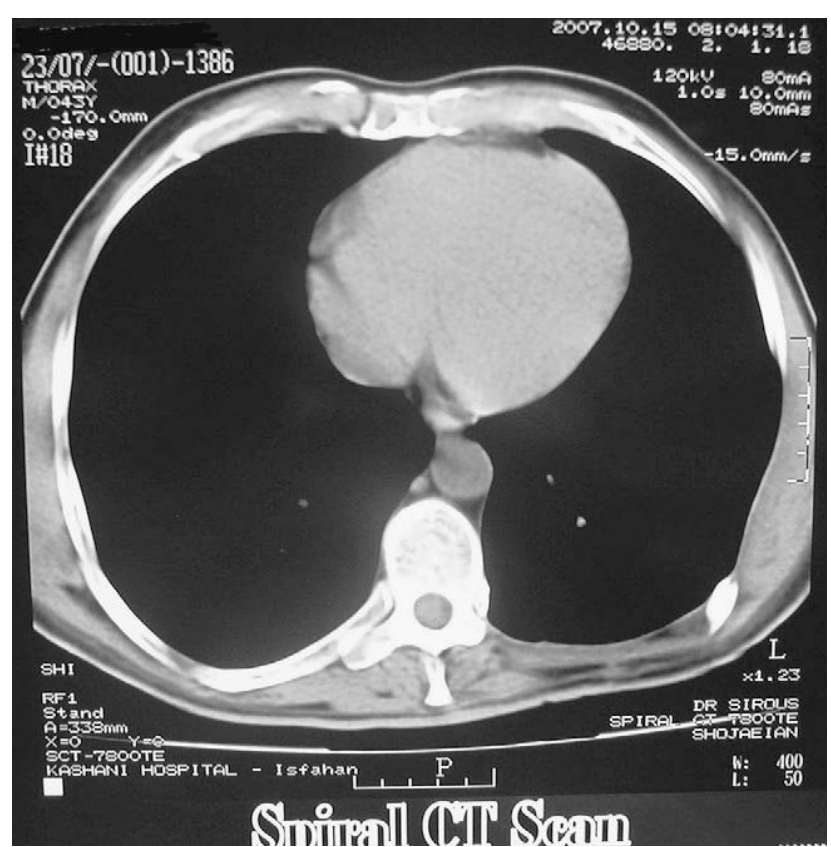

FIGURE 4. Follow-up study 7 months later shows no evidence of tumor residue or recurrence.

with a few being documented in Western countries. ${ }^{8}$ The patients are referred to medical centers for chest pain, dyspnea, and constitutional symptoms such as fever or recent weight loss. Pyothorax-associated lymphoma occurs in $2 \%$ of patients between 22 and 55 years after the onset of tuberculosis. The mean age of patients is 51.8 years with a male predominance of 5:2., ${ }^{3,9}$ The absolute mechanism of pyothorax-associated lymphoma is not detected yet. Chronic stimulation of $\mathrm{B}$ cells results in production of growth factors such as interleukin 6 and interleukin 10, which play important roles in neoplastic cell growth. ${ }^{3,7,8}$ Other mechanisms that are considered for primary pleural lymphoma are antecedent autoimmune disease and Epstein-Barr virus infection. ${ }^{3}$ Our first patient has received immunosuppressive therapy for kidney transplantation, which can be a mechanism for his disease.

Despite the prevalence of tuberculous in Iran, no pyothorax-associated lymphoma is documented yet, because artificial pneumothorax therapy is not performed as a routine treatment. Our first patient had a primary pleural lymphoma without a history of tuberculous pyothorax or artificial pneumothorax as a treatment for lung tuberculosis. Primary pleural lymphoma without chronic pyothorax is a very uncommon tumor. According to previous studies, our patient is the tenth case reported since $1996 .^{3}$

The diagnosis and treatment of pleural lymphoma is controversial. Previous study indicated that fine needle aspiration or nonexcisional biopsy as a diagnostic modality is not appropriate because of wrong diagnosis owing to inade- quate tissue specimen and the risk of tumor implantation. ${ }^{2,5,10}$ According to some suggestions, all primary chest wall tumors should be subjected to at least excisional biopsy, although highly suspected malignancies should receive wide radical resection or subsequent resection for safe margins. ${ }^{1,2,5}$ Nonexcision biopsy should be done only in patients with suspicion of metastasis or hematologic disease, who do not benefit from aggressive surgical resection. ${ }^{1,2,5,11}$ In patient 1 , excisional biopsy was done for diagnosis and then chemotherapy was used. The patient was in complete remission after about 5 months' followup. Because of the rarity of cases, the treatment has not been definitely determined. Although the primary treatment for lymphoma is chemotherapy, surgery followed by adjuvant chemotherapy can provide a satisfactory outcome in patients in whom the chest wall lymphoma was the only site of disease. $^{5}$

Our second patient had a primary non-Hodgkin lymphoma in the ribs. Primary lymphoma of the bone (PLB), which was first reported by Oberling, ${ }^{12}$ now is recognized as an independent clinicophathologic entity. ${ }^{4,13}$ PLB accounts for approximately $4.7 \%$ of extranodal lymphomas. ${ }^{14}$ The majority of cases occur in long bones, pelvis, or spine, and rib involvement is rare $(2 \%$ to $15 \%$ of cases of PLB). ${ }^{4,13}$ The mean age at presentation for PLB, according to a review by Ostrowski and associates, ${ }^{15}$ was 46 years with male predominance. The most frequent symptoms are pain, swelling, and B symptoms (fever, night sweats, and body weight loss), as in other lymphoma conditions. ${ }^{4,16}$ Because imaging study of chest wall tumor suggesting PLB is not diagnostic, a histologic specimen is needed. ${ }^{2,5,17}$ The treatment in general is conservative, based on the stage of disease. There is no definite therapeutic scheme. Local radiation is recommended, and additional systemic chemotherapy seems to be effective. ${ }^{16}$

As discussed earlier, we chose surgery for histologic diagnosis. The left eighth rib and the original mass were completely resected in our patient. The diagnosis was performed by histology and confirmed by immunophenotyping. After radical resection, we prescribed chemotherapy for the patient. The patient has been in complete remission for more than 8 months since the operation.

\section{References}

1. Incarbone M, Pastorino U. Surgical treatment of chest wall tumors. World J Surg. 2001;25:218-30.

2. Hsu PK, Hsu HS, Lee HC, Hsieh CC, Wu YC, Wang LS, et al. Management of primary chest wall tumors: 14 years' clinical experience. J Chin Med Assoc. 2006;69:377-82.

3. Hirai S, Hamanaka Y, Mitsui N, Morifuji K, Sutoh M. Primary malignant lymphoma arising in the pleura without preceding long-standing pyothorax. Ann Thorac Cardiovasc Surg. 2004;10:297-300.

4. Nishiyama N, Nakatani S, Inoue K, Katoh T, Kinoshita H. Primary lymphoma of bone originating in a rib. Jpn J Thorac Cardiovasc Surg. 2000;48:180-3.

5. Hsu PK, Hsu HS, Li AF, Wang LS, Huang BS, Huang MH, et al. Non-Hodgkin's lymphoma presenting as a large chest wall mass. Ann Thorac Surg. 2006;81: 1214-8. 
6. King RM, Pairolero PC, Trastek VF, Piehler JM, Payne WS, Bernatz PE. Primary chest wall tumors: factors affecting survival. Ann Thorac Surg. 1986;41:597-601.

7. Molinie V, Pouchot J, Navratil E, Aubert F, Vinceneux P, Barge J. Primary Epstein-Barr virus-related non-Hodgkin's lymphoma of the pleural cavity following long-standing tuberculous empyema. Arch Pathol Lab Med. 1996; 120:288-91.

8. Androulaki A, Drakos E, Hatzianastassiou D, Vgenopoulou S, Gazouli M, Korkolopoulou P, et al. Pyothorax-associated lymphoma (PAL): a western case with marked angiocentricity and review of the literature. Histopathology. 2004; 44:69-76.

9. Fukayama M, Ibuka T, Hayashi Y, Ooba T, Koike M, Mizutani S. Epstein-Barr virus in pyothorax-associated pleural lymphoma. Am J Pathol. 1993;143:1044-9.

10. Maitra A, Timmons CF, Siddiqui MT, Saboorian MH. Fine-needle aspiration biopsy features in a case of giant cell fibroblastoma of the chest wall. Arch Pathol Lab Med. 2001;125:1091-4.
11. Pinto RG, Mandreker S, Vernekar JA. Multiple myeloma presenting as a subcutaneous nodule on the chest wall: diagnosis by fine needle aspiration. Acta Cytol. 1997;41:1233-4.

12. Oberling C. Les réticulosarcomes et les réticulo-endothéliosarcomes de la moelle osseuse (sarcomes d'Ewing). Bull Assoc Fr Cancer. 1928;17:259-63.

13. Ueda T, Aozasa K, Ohsawa M, Yoshikawa H, Uchida A, Ono K, et al. Malignant lymphomas of bone in Japan. Cancer. 1989;64:2387-92.

14. Freeman C, Berg JW, Cutler SJ. Occurrence and prognosis of extranodal lymphomas. Cancer. 1972;29:252-60.

15. Ostrowski ML, Unni KK, Banks PM, Shives TC, Evans RG, O’Connell MJ, et al. Malignant lymphoma of bone. Cancer. 1986;58:2646-55.

16. Durr HR, Muller PE, Hiller E, Maier M, Baur A, Jansson V, et al. Malignant lymphoma of bone. Arch Orthop Trauma Surg. 2002;122:10-6.

17. Doll C, Wulff B, Rossler J, Schaper J, Havers W. Primary B-cell lymphoma of bone in children. Eur J Pediatr. 2001;160:239-42. 